\title{
A Geometric view of Magnetic Surfaces and Magnetic Curves
}

\author{
Zehra Özdemir*, İsmail Gök and F. Nejat Ekmekci \\ (Communicated by Kazım İlarslan)
}

\begin{abstract}
In the present paper, we approach the magnetic surfaces geometrically. For this aim, we study the problem of constructing a family of magnetic surfaces from a given magnetic field line. We obtain a parametric representation for the surfaces family whose members share the same magnetic field lines and magnetic curves. We investigate the trajectory of charged particles moving related to these surfaces. Moreover, we give various examples of these surfaces and illustrate their figures.
\end{abstract}

Keywords: Magnetic vector fields; magnetic curve; special surfaces.

AMS Subject Classification (2010): 76X05, 53A04, 65D17.

\section{Introduction}

A vector field with zero divergences is called a magnetic field. A magnetic surface defined as a surface in which the magnetic field lines lie. The best example we can give to the magnetic surface is plasma. A plasma is a hot ionized gas consisting of approximately equal numbers of positively charged ions and negatively charged electrons. The characteristics of plasmas are significantly different from those of ordinary neutral gases so that plasmas are considered a distinct "fourth state of matter. Most of the universe is in the form of a plasma with a magnetic field perforated. The magnetic field breaks and reconnects quickly when the lines are bent or cut, transforming the magnetic energy into heat, kinetic energy, and fast particle energy. As a result of these situations, the magnetic surfaces for the related magnetic field lines can obtain in different forms. These surfaces can be smooth or non-smooth. The most common example of magnetic surfaces is flux tubes. As used in astrophysics, a flux tube generally has a larger magnetic field and other properties that differ from the surrounding space. These flux tubes commonly find around many stars and the sun. Some planets also have flux tubes. A well-known example is the flux tube between Jupiter and its moon io $[4,5,9,10]$. Penning has developed a model called Penning trap to study the motion of charged particles and confinement of plasma. This model has been one of the most successful models and has contributed greatly to work done in this area. However, this model can be used for the confinement of single species plasmas. Later, the importance of magnetic surfaces has recognized in magnetic fusion research. For the first time, Pedersen et al. have shown that non-neutral plasmas can be confined to magnetic surfaces. Magnetic surface configurations, such as stellarators and tokamaks, are highly developed and studied in the context of thermonuclear fusion, and have recently become of interest for the confinement of non-neutral plasmas. They have certain advantages over open and closed field line systems, such as the Penning trap. Magnetic surface configurations confine both positive and negative species simultaneously, at any level of charge imbalance from pure electron to quasineutral. They may provide stabilization of diocotron modes, and confine energetic electrons and positrons at modest magnetic field strengths. Hence, such configurations have unique advantages for confine nonneutral electron-ion plasmas and antiproton positron plasmas in the positron-electron laboratory [10].

Received : 01-November-2018, Accepted : 01-February-2019

* Corresponding author

This article is the written version of authors plenary talk delivered on August 28-31, 2018 at 7th International Eurasian Conference on Mathematical Sciences and Applications (IECMSA-2018) at Kyiv-Ukraine 
One of the most important applications of the magnetic surface is the creation of the first limited laboratory positron-electron plasma. Configurations with magnetic surfaces also have the additional advantage that owing to the small mass, energetic positrons or electrons can be confined at rather modest magnetic field strengths.

On the other hand, magnetic curves have many applications in physics and differential geometry and play an important role in these fields. When a charged particle enters a magnetic field, the particle's Serret-Frenet vectors are influenced by this field, resulting in a force called the Lorentz force. With the effect of this force, the particle follows a new trajectory within this field. This trajectory is called a magnetic curve. Lorentz force equation associated with the magnetic field given as

$$
\phi(X)=V \times X
$$

and the trajectories associated with the magnetic vector field $V$ is given by

$$
\nabla_{T} T=\phi(T)=V \times T
$$

With this equation, we studied that the magnetic field in space has non-zero curvature. So, this gives a more important and real approach than the classical approach. Also, this formula and the Hall effect (which explains the dynamics of an electric current flow $X$ in $\mathbb{R}^{3}$ when exposed to a perpendicular magnetic field $V$ ) give important applications to us in analytical chemistry, biochemistry, atmospheric science, geochemistry, cyclotron, proton, cancer therapy, and velocity selector.(see for details $[1,2,3,6,7,8]$ ).

The article shaped as follows: In Section 2, we give some basic concepts that we use in the article. The parametric representation and images of the magnetic surfaces are obtained. In Section 3, a parametric representation for a magnetic surface pencil that shares the same magnetic trajectory as an isoparametric curve is derived with some illustrated examples. In Section 4, we obtain the relation between the magnetic curves and magnetic surfaces pencil.

\section{Preliminaries}

Divergence free vector field called as a magnetic field. The magnetic field lines satisfy the following equations

$$
\frac{\partial x}{\partial s}=B_{x}, \frac{\partial y}{\partial s}=B_{y}, \frac{\partial z}{\partial s}=B_{z} \text { or } \frac{\partial r}{\partial s}=B
$$

Let $C: r=r(s), 0<s<I$ be parametric representation for magnetic field lines and $T(s), N(s), B(s)$ be tangent, principle normal and binormal vectors of the curve $C$, respectively. Then the parametric equation of the magnetic surface can be given as follows:

$$
P:[0, I] \times[0, T] \rightarrow \mathbb{R}^{3}: P(s, t)=r(s)+u(s, t) T(s)+v(s, t) N(s)+w(s, t) B(s),
$$

where $u(s, t), v(s, t)$ and $w(s, t)$ are all $C^{1}$ functions.

Since the magnetic field line $C$ is an isoparametric curve on the surface $P(s, t)$, there exist a parameter $t_{0} \in[0, T]$ such that $P\left(s, t_{0}\right)=r(s), 0 \leq s \leq I$, that is, $u\left(s, t_{0}\right)=v\left(s, t_{0}\right)=w\left(s, t_{0}\right)=0,0<s<I, 0<t_{0}<T$. For the case when the functions $u, v$ ve $w$ depend only on the parameter $t$; the isogeodesic surface pencil becomes

$$
P(s, t)=r(s)+u(t) T(s)+v(t) N(s)+w(t) B(s)
$$

[11]. Some surfaces corresponding to special cases of $u(s, t), v(s, t)$ and $w(s, t)$ are given below.

i. If $u(s, t)=0, v(s, t)=r \cos t$ and $w(s, t)=r \sin t$ then the surface $P(s, t)$ is called as magnetic tube surface.

ii. If $u(s, t)=0, v(s, t)=r(s, t) \cos t$ and $w(s, t)=r(s, t) \sin t$ the surface $P(s, t)$ is called as generalized magnetic tube surface.

iii. If $u(s, t)=0, v(s, t)=\exp [\alpha s] b \cos t$ and $w(s, t)=\exp [\alpha s] b \sin t$ then the surface $P(s, t)$ is called as magnetic seashell surfaces.

The magnetic surfaces defined in this way also found in biological structures such as a seashell, horn, and snail shell. The best example of this derived by the Illert [12]. He gives an elegant logically complete and physically meaningful optimization problem for seashell Spiers and growth trajectories. We obtain some examples of magnetic surfaces including some biological structures. 


\subsection{Examples of magnetic surfaces}

Example 2.1. Let $B_{x}=-x+y, B_{y}=-x+2 y-2 z, B_{z}=2 y-z$ be the components of the magnetic field $B$. The functions $P(x, y, z), Q(x, y, z), R(x, y, z)$ are defined in a $D$ region of $x y z$ space and the functions can be derived continuously from the first order by independent variables. Considering the magnetic field line satisfy the following first order system of linear differential equation.

$$
\frac{\partial x(s)}{\partial s}=-x(s)+y(s), \frac{\partial y(s)}{\partial s}=-x(s)+2 y(s)-2 z(s), \frac{\partial z(s)}{\partial s}=2 y(s)-z(s) .
$$

Let us assume that the solution of the given equation is a surface family of solutions $z=f(x, y)$. The vector $N=(x, y, z)$ is a vector perpendicular to this surface family. Since the last equation can also be written as

$$
P(x, y, z) z_{x}+Q(x, y, z) z_{y}-R(x, y, z)=0
$$

the vector $M=(P, Q, R)$ becomes a tangent vector to the surface family $z=f(x, y)$. The vector $M=(P, Q, R)$ is perpendicular to the vector $N=\left(z_{x}, z_{y},-1\right)$, so this vector will be parallel to the tangent vector of a curve $\gamma$ on the surface. If the curve $\gamma$ is given by the vector equation $V=(x, y, z)$ in $x y z$ space, the tangent vectors are given by $T=(d x, d y, d z)$. In this case, the vector $M=(P, Q, R)$ is parallel to the vector $T$. Thus the following relation is obtained for the given differential equation.

$$
\frac{d x}{-x+y}=\frac{d y}{-x+2 y-2 z}=\frac{d z}{2 y-z} \text {. }
$$

The general solution $F(u, v)=0$ of the obtained system is given as follows, where $u(x, y, z)=c_{1}$ and $v(x, y, z)=$ $c_{2}$ are independent solutions of the last differential equation systems

$$
\gamma(s)=\left(\begin{array}{c}
\frac{2}{\sqrt{11}} e^{s / 2} \sin \frac{\sqrt{11}}{2} s+\frac{6}{55} e^{-s}\left(-11+11 e^{3 s / 2} \cos \frac{\sqrt{11}}{2}-3 \sqrt{11} e^{3 s / 2} \sin \frac{\sqrt{11}}{2}\right) \\
+\frac{2}{55} e^{-s}\left(44+11 e^{3 s / 2} \cos \frac{\sqrt{11}}{2}-3 \sqrt{11} e^{3 s / 2} \sin \frac{\sqrt{11}}{2} s\right), \\
-\frac{16}{\sqrt{11}} e^{s / 2} \sin \frac{\sqrt{11}}{2}+\frac{2}{\sqrt{11}} e^{s / 2}\left(11 \cos \frac{\sqrt{11}}{2}+3 \sqrt{11} \sin \frac{\sqrt{11}}{2} s\right), \\
\frac{8}{\sqrt{11}} e^{s / 2} \sin \frac{\sqrt{11}}{2} s+\frac{3}{55} e^{-s}\left(11+44 e^{3 s / 2} \cos \frac{\sqrt{11}}{2}-12 \sqrt{11} e^{3 s / 2} \sin \frac{\sqrt{11}}{2}\right) \\
+\frac{4}{55} e^{-s}\left(-11+11 e^{3 s / 2} \cos \frac{\sqrt{11}}{2}-3 \sqrt{11} e^{3 s / 2} \sin \frac{\sqrt{11}}{2} s\right)
\end{array}\right)
$$

Then, the magnetic surface related to the magnetic field line $\gamma$ given in Figure 1.

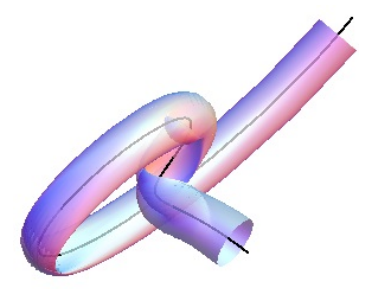

Figure 1. Magnetic surfaces $u(s, t)=2 \sin t \cos s, v(s, t)=2 \sin t \sin s, w(s, t)=2 \cos t$.

Example 2.2. In three dimension the components of the magnetic field lines are given as follows

$$
x^{\prime}=x+\sqrt{2} y, y^{\prime}=-\sqrt{2} x-y, z^{\prime}=1
$$

the solution of this equation is calculated as

$$
\gamma(s)=\left(\begin{array}{c}
\frac{(-1+\sqrt{-3}) e^{-\sqrt{-3} s}+(1+\sqrt{-3}) e^{\sqrt{-3} s}}{2 \sqrt{-3}}+\frac{-e^{-\sqrt{-3} s}+e^{\sqrt{-3} s}}{\sqrt{-3}}, \\
\frac{(1+\sqrt{-3}) e^{-\sqrt{-3} s}+(-1+\sqrt{-3}) e^{\sqrt{-3} s}}{2 \sqrt{-3}}-\frac{-e^{-\sqrt{-3} s}+e^{\sqrt{-3} s}}{\sqrt{-3}} \\
s+1
\end{array}\right)
$$

Then the surface related to the curve $\gamma$ are given in Figure 2: 


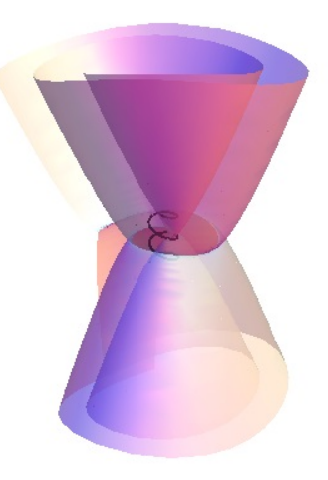

Figure 2. Magnetic surfaces $u(s, t)=t, v(s, t)=t^{2}-2 s, w(s, t)=t^{3}+s$.

Example 2.3. The (generalized) tube surface corresponding to the magnetic field $B(-x+y,-x+2 y-2 z, 2 y-z)$ is given in Figure 3.

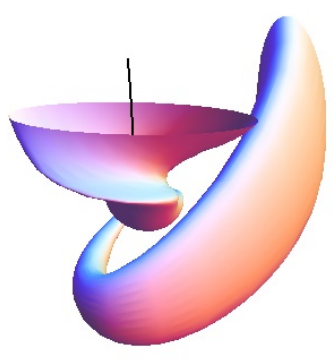

Figure 3. Magnetic tube surface $u(s, t)=0, v(s, t)=s \cos t, w(s, t)=s^{2} \sin t$.

Example 2.4. The shell surfaces sea surfaces corresponding to the magnetic field $B(-x+y,-x+2 y-2 z$, $2 y-z)$ and $(x+\sqrt{2} y,-\sqrt{2} x-y, 1)$ are given in Figure 4 and Figure 5.
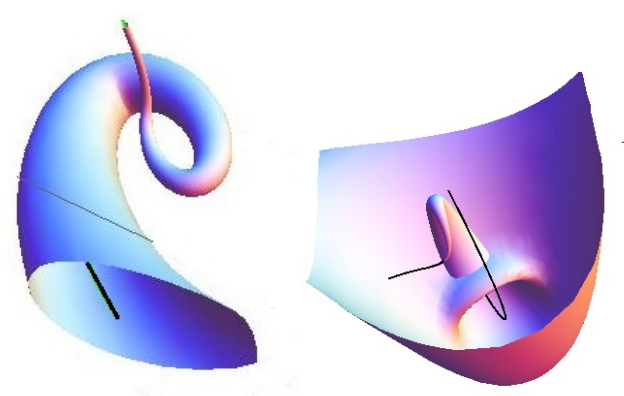


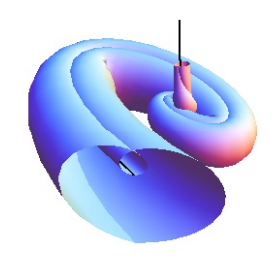

Figure 4. Seashell magnetic surfaces $(\mathrm{a}) \mathrm{u}(\mathrm{s}, \mathrm{t})=0, \mathrm{v}(\mathrm{s}, \mathrm{t})=\mathrm{e}^{s / 2} \sin t, w(s, t)=e^{s / 2} \cos t,(b) u(s, t)=0, v(s, t)=e^{s} \cos t, w(s, t)=e^{s} \sin t,(c) u(s, t)=$ $0, v(s, t)=t e^{s / 4} \sin t$

Figure 5. Seashell magnetic surfaces (a) $\quad \mathrm{w}(\mathrm{s}, \mathrm{t}) \quad=\mathrm{te}^{s / 4} \cos t, u(s, t)=0, v(s, t)=e^{s / 2} \sin t, w(s, t)=e^{s / 2} \cos t,(b) u(s, t)=0, v(s, t)=$ $s e^{s} \sin t, w(s, t)=s e^{s}$.
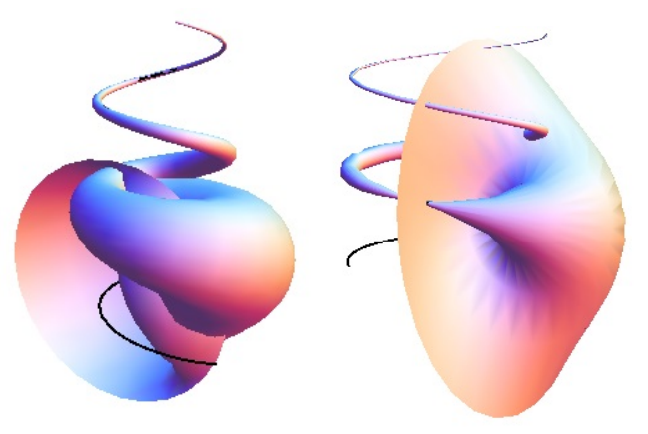

In this section, the problem of forming a magnetic surface family using a specific magnetic curve investigated. The aim is to provide a parametric representation for a magnetic surface pencil that shares the same magnetic trajectory as an isoparametric curve.

\section{Magnetic surfaces with common magnetic curves}

Proposition 3.1. Let $\gamma$ be a unit speed magnetic curve on the surfaces $P(s, t)$ with the Darboux frame $\{t, y, n\}$. Then Lorentz force equation of the Darboux frame written as

$$
\left[\begin{array}{l}
\phi(t) \\
\phi(y) \\
\phi(n)
\end{array}\right]=\left[\begin{array}{ccc}
0 & k_{g} & k_{n} \\
-k_{g} & 0 & \varpi \\
-k_{n} & -\varpi & 0
\end{array}\right]\left[\begin{array}{l}
t \\
y \\
n
\end{array}\right]
$$

where $\varpi$ is a certain function defined by $\varpi=g(\phi(y), n)$.

Proof. Let $\gamma$ be a unit speed magnetic curve on the surfaces $P(s, t)$ with the Darboux frame $\{t, y, n\}$. From the definition of the magnetic curve we know that

$$
\phi(t)=\nabla_{t} t=k_{g} y+k_{n} n .
$$

Since $\phi(y) \in \operatorname{span}\{t, y, n\}$, we have

$$
\phi(y)=\delta t+\lambda y+\mu n
$$

Then, the equalities

$$
\begin{aligned}
& \delta=g(\phi(y), t)=-g(\phi(t), y)=-k_{g} \\
& \lambda=g(\phi(y), y)=0 \\
& \mu=g(\phi(y), n)=\varpi
\end{aligned}
$$


give us

Similarly, we can easily obtain that

$$
\phi(y)=-k_{g} t+\varpi n
$$

$$
\phi(n)=-k_{n} t-\varpi n .
$$

Proposition 3.2. Let $\gamma$ be a unit speed curve on the surfaces $P(s, t)$. Then the curve $\gamma$ is a magnetic trajectory of a magnetic field $B$ if and only if the vector field $B$ can be written along the curve $\gamma$ as

$$
B=\varpi t-k_{n} y+k_{g} n \text {. }
$$

Proof. Let $\gamma$ be a unit speed magnetic trajectory of a magnetic field $B$. Then using Proposition 3.1, we can easily see that

$$
B=\varpi t-k_{n} y+k_{g} n
$$

Conversely, we assume that the Eq.(3.2) holds. Then we get $B \times t=\phi(t)$. So, the curve $\gamma$ is a magnetic trajectory of the magnetic vector field $B$.

Now we can give the following theorem and corollary.

Theorem 3.1. Let $\gamma$ be a magnetic trajectory of a magnetic field $B$ and $M$ be a magnetic surface associated with the magnetic field $B$. Then magnetic curves are geodesics on the magnetic surface $M$

Proof. Let $\gamma$ be a unit speed curve on the surfaces $M$. If $M$ is a magnetic surface of magnetic curve $\gamma$ then $g\left(\frac{\partial r}{\partial s}, n\right)=k_{g}=0$. This implies that magnetic curves are geodesic curves on the magnetic surface $M$.

Corollary 3.1. (Main result) Suppose we are given a spatial parametric curve $r: r(s) \subset \mathbb{R}^{3}$ in which $s$ is the arc length, and $r(s)$ has third derivatives. The parametric surface $P(s, t)$ is defined based on the given curve $r(s)$. Then the curve $r$ is an isomagnetic curve on the surface $P(s, t)$ if and only if the functions $u, v$ ve $w$ depend only on the parameter $t$

$$
P(s, t)=r(s)+u(t) T(s)+v(t) N(s)+w(t) B(s) .
$$

Proof. Let $C: r=r(s), 0<s<I$, be parametric representation of magnetic field lines. Then the parametric form of the surfaces pencils $P(s, t)$ given as

$$
P(s, t)=r(s)+u(s, t) T(s)+v(s, t) N(s)+w(s, t) B(s), 0<s<I, 0<t<T
$$

where $u(s, t), v(s, t)$ and $w(s, t)$ are all $C^{1}$ functions. The normal vector of the surface $P(s, t)$ can be calculated by cross product of the partial differentiations; That is, based on the following derivation using the Serret-Frenet formula:

$$
\begin{aligned}
& \frac{\partial P(s, t)}{\partial s}=\left(1+u_{s}-\varkappa v\right) T(s)+\left(v_{s}+\varkappa u-\tau w\right) N(s)+(w+\tau v) B(s), \\
& \frac{\partial P(s, t)}{\partial t}=u_{t} T(s)+v_{t} N(s)+w_{t} B(s) .
\end{aligned}
$$

If we apply isoparametric conditions, the normal vector can be expressed as

$$
n(s, t)=\phi_{1} T(s)+\phi_{2} N(s)+\phi_{3} B(s),
$$

where

$$
\begin{aligned}
\phi_{1} & =w_{t} v_{s}-v_{t} w_{s} \\
\phi_{2} & =u_{t} w_{s}-w_{t}\left(1+u_{s}\right) \\
\phi_{3} & =v_{t}\left(1+u_{s}\right)-u_{t} v_{s}
\end{aligned}
$$

Then using the following equation

$$
g\left(\frac{\partial r}{\partial s}, n\right)=0
$$

we have, for the case when the marching-scale functions depend only on the parameter $t$ the magnetic isogeodesic surface pencil becomes

$$
P(s, t)=r(s)+u(t) T(s)+v(t) N(s)+w(t) B(s) .
$$


In this section, we illustrate that it is possible to derive exact magnetic surface pencils by specifying a magnetic curve that represents a geometric feature of the object to design. We present some concrete examples to illustrate this viewpoint.

3.0.1. Examples In this first example, we construct a magnetic surface pencil in which all the surfaces share a magnetic trajectory related to the magnetic vector field $B=(-x+y,-x+2 y-2 z, 2 y-z)$.

Example 3.1. The magnetic surfaces related to the magnetic field $B$ are given in Figure 6-8.

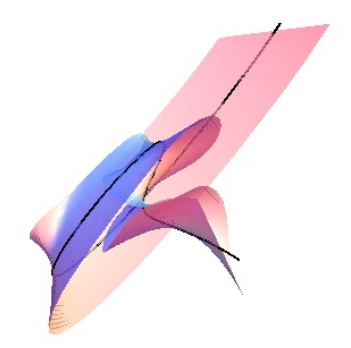

Figure 6. Magnetic surfaces and magnetic curve $u(s, t)=v(s, t)=w(s, t)=t$.

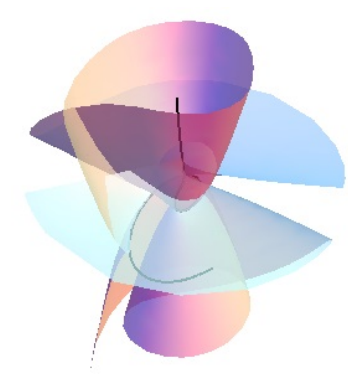

Figure 7. Magnetic surface and magnetic curve $\mathrm{u}(\mathrm{s}, \mathrm{t})=\mathrm{t}, \mathrm{v}(\mathrm{s}, \mathrm{t})=\mathrm{t}^{2}, w(s, t)=t^{3}$.

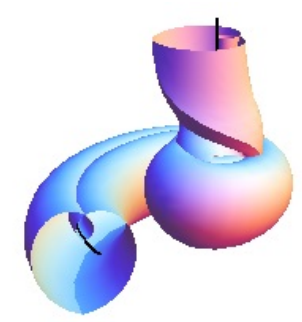

Figure 8. Magnetic tube surfaces and magnetic curve. $\mathrm{u}(\mathrm{s}, \mathrm{t})=0, \mathrm{v}(\mathrm{s}, \mathrm{t})=\mathrm{t} \cos t, w(s, t)=t \sin t$.

Now, we construct a magnetic surface pencil in which all the surfaces share a magnetic trajectory related to the magnetic vector field $B=(x+\sqrt{2} y,-\sqrt{2} x-y, 1)$.

Example 3.2. Then the surface related to the magnetic field $B$ is given in Figure 9. 


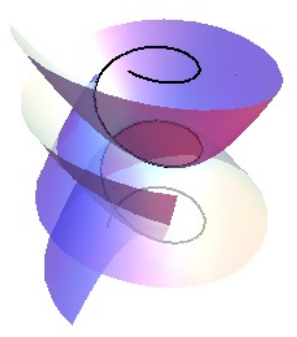

Figure 9. Magnetic surfaces and magnetic curve $\mathrm{u}(\mathrm{s}, \mathrm{t})=\mathrm{t}, \mathrm{v}(\mathrm{s}, \mathrm{t})=\mathrm{t}^{2}, w(s, t)=t^{3}$.

\section{Magnetic Curves Related to the Magnetic Surfaces Pencil}

In this section we investigate the magnetic curves related to the magnetic field lines $B$.

Theorem 4.1. Let $\gamma: \gamma(s)=(x(s), y(s), z(s)) \subset \mathbb{R}^{3}$ be an arclength parameterized curve and $B=\left(B_{x}, B_{y}, B_{z}\right)$ be a magnetic field. Then the curve $\gamma$ is a magnetic curve associated with the magnetic field $B$ iff the magnetic curves parameterized by

$$
\begin{aligned}
x(s) & =\int \cos \theta(s) \cos \varphi(s) d s, \\
y(s) & =\int \cos \theta(s) \sin \varphi(s) d s, \\
z(s) & =\int \sin \theta(s) d s .
\end{aligned}
$$

Where $\theta$ and $\varphi$ satisfy the following differential equation

$$
B_{x}\left(-\theta^{\prime} \sin \theta \cos \varphi-\varphi^{\prime} \cos \theta \sin \varphi\right)+B_{y}\left(-\theta^{\prime} \sin \theta \sin \varphi+\varphi^{\prime} \cos \theta \cos \varphi\right)+B_{z}\left(\theta^{\prime} \cos \theta\right)=0 .
$$

Proof. If $\gamma(s)=(x(s), y(s), z(s))$ is a magnetic curve of the magnetic field $B$ then $\gamma$ satisfy

$$
\nabla_{\gamma^{\prime}} \gamma^{\prime}=B \times \gamma^{\prime} .
$$

Using the last equation we get following differential equation

$$
\begin{aligned}
& x^{\prime \prime}=B_{y} z^{\prime}-B_{z} y^{\prime}, \\
& y^{\prime \prime}=B_{z} x^{\prime}-B_{x} z^{\prime}, \\
& z^{\prime \prime}=B_{x} y^{\prime}-B_{y} x^{\prime} .
\end{aligned}
$$

From the first two equations we have

$$
-B_{x} B_{z} y^{\prime}+B_{y} B_{z} x^{\prime}=B_{x} x^{\prime \prime}+B_{y} y^{\prime \prime} .
$$

If we use the last equation we obtain

$$
B_{x} x^{\prime \prime}+B_{y} y^{\prime \prime}+B_{z} z^{\prime \prime}=0 .
$$

On the other hand, we know that $\gamma$ is a unit speed curve, that is, $x^{\prime}(s)^{2}+y^{\prime}(s)^{2}+z^{\prime}(s)^{2}=1$. Then using the spherical coordinates we have

$$
\begin{aligned}
x^{\prime}(s) & =\cos \theta(s) \cos \varphi(s), \\
y^{\prime}(s) & =\cos \theta(s) \sin \varphi(s), \\
z^{\prime}(s) & =\sin \theta(s)
\end{aligned}
$$

This implies,

$$
B_{x}\left(-\theta^{\prime} \sin \theta \cos \varphi-\varphi^{\prime} \cos \theta \sin \varphi\right)+B_{y}\left(-\theta^{\prime} \sin \theta \sin \varphi+\varphi^{\prime} \cos \theta \cos \varphi\right)+B_{z}\left(\theta^{\prime} \cos \theta\right)=0 .
$$


In the following example, the trajectory of a charged particle moving within a magnetic surface bounding the magnetic field plotted.

Example 4.1. The particle moves a helical trajectory (magnetic curve) along the magnetic field lines in the magnetic surfaces are given in Figure 10.

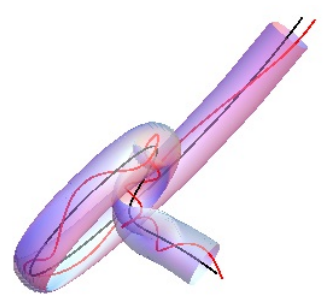

Figure 10. Magnetic curve(Red) in the magnetic surface $\mathrm{u}(\mathrm{s}, \mathrm{t})=2 \sin t \cos s, v(s, t)=2 \sin t \sin s, w(s, t)=2 \cos t$.

\section{References}

[1] Barros, M., Romero, A., Magnetic vortices. EPL, 77(2007) 1-5.

[2] Barros, M., Cabrerizo, J.L., Fernandez,M., Romero, A., Magnetic vortex filament flows. J. Math. Phys.,48(8) (2007) 082904.

[3] Barros, M. General helices and a theorem of Lancret. Proc. Am.Math. Soc.,125, 1503-1509, 1997.

[4] Bird, B.R., Stewart, W.E., Lightfoot, E. N., Transport Phenomena. Wiley. ISBN 0-471-07392-X, 1960.

[5] Boozer, A.H., Physics of magnetically confined plasmas. Rev. Mod. Phys., DOI:https://doi.org/10.1103/RevModPhys.76.1071, 2005.

[6] Bozkurt, Z.,Gök, İ., Yaylı Y., Ekmekci, F.N., A new Approach for Magnetic Curves in Riemannian 3D-Manifolds. J. Math. Phys., 55(2014), $1-12$.

[7] Cabrerizo, J.L., Magnetic fields in 2D and 3D sphere. J. Nonlinear Math. Phys., 20(3)(2013), 440-4503.

[8] Druţ-Romaniuc, S.L., Munteanu, M.I., Magnetic curves corresponding to Killing magnetic fields in E3. J. Math. Phys.,52(2011), 113506,

[9] Hazeltine, R.D., Meiss, J. D. Plasma Confinement. Dover publications. inc. Mineola, New York, 2003.

[10] Pedersen, T.S., Boozer, A.H., Confinement of nonneutral plasmas on magnetic surfaces. Phys. Rev. Lett., 88 (2002), 205002.

[11] Wang, G.J., Tang, K., Tai, C.L., Parametric representation of a surface pencil with a common spatial geodesic. Computer-Aided Design, 36(5)(2004), 447-459.

[12] Illert, C., Formulation and solution of the classical problem, II Tubular three dimensional surfaces. Nuovo Cimento, 11(1989), 761-780.

\section{Affiliations}

\section{ZEHRA ÖZDEMIR}

AdDREss: Amasya University, Dept. of Mathematics, 05000, Amasya-Turkey

E-MAIL: zehra.ozdemir@amasya.edu.tr

ORCID ID: orcid.org/0000-0001-9750-507X

İsMAIL GÖK

ADDRESs: Ankara University, Dept. of Mathematics, 06100, Ankara-Turkey

E-MAIL: igok@science.ankara.edu.tr

ORCID ID: orcid.org/0000-0001-8407-133X

\section{F. NEJAT EKMEKCI}

ADDREsS: Ankara University, Dept. of Mathematics, 06100, Ankara-Turkey

E-MAIL: ekmekci@science.ankara.edu.tr

ORCID ID: orcid.org/0000-0003-1246-2395 